\title{
Telmisartan Protects Auditory Hair Cells from Gentamicin-Induced Toxicity in vitro
}

\author{
Maurizio Cortada ${ }^{a}$ Eric Wei ${ }^{a}$ Neha Jain ${ }^{a}$ Soledad Levano ${ }^{a}$ Daniel Bodmer ${ }^{b}$ \\ ${ }^{a}$ Department of Biomedicine, University of Basel, Basel, Switzerland; ${ }^{b}$ Clinic for Otolaryngology, Head and \\ Neck Surgery, University of Basel Hospital, Basel, Switzerland
}

\section{Keywords}

Cochlea · Gentamicin toxicity · GW9662 · Hair cells · Inner ear. Organ of Corti · Peroxisome proliferator-activated receptor $\gamma \cdot$ Telmisartan

\begin{abstract}
Background: Telmisartan is an angiotensin II receptor blocker that has pleiotropic effects and protective properties in different cell types. Moreover, telmisartan has also shown partial agonism on the peroxisome proliferator-activated receptor $\gamma$ (PPAR- $\gamma)$. Auditory hair cells (HCs) express PPAR- $\gamma$, and the protective role of PPAR- $\gamma$ agonists on $\mathrm{HCs}$ has been shown. Objectives: The objective of this study was to investigate the effects of telmisartan on gentamicin-induced ototoxicity in vitro. Methods: Cochlear explants were exposed to gentamicin with or without telmisartan, and/or GW9662, an irreversible PPAR- $\gamma$ antagonist. Results: Telmisartan protected auditory HCs against gentamicin-induced ototoxicity. GW9662 completely blocked this protective effect, suggesting that it was mediated by PPAR- $\gamma$ signaling. Exposure to GW9662 or telmisartan alone was not toxic to auditory HCs. Conclusions: We found that telmisartan, via PPAR- $\gamma$ signaling, protects auditory $\mathrm{HCs}$ from gentamicin-induced ototoxicity. Therefore, telmisartan could potentially be used in the future to prevent or treat sensorineural hearing loss.
\end{abstract}

(c) 2020 The Author(s)

Published by S. Karger AG, Basel karger@karger.com www.karger.com/aud

Karger $\stackrel{\text { ' }}{5}$

GOPEN ACCESS
(C) 2020 The Author(s)

Published by S. Karger AG, Basel

This article is licensed under the Creative Commons AttributionNC-ND) (http://www.karger.com/Services/OpenAccessLicense) Usage and distribution for commercial purposes as well as any disNonCommercial-NoDerivatives 4.0 International License (CC BYtribution of modified material requires written permission.

\section{Introduction}

There are 466 million people worldwide who suffer from disabling hearing loss [WHO, 2018] and adult onset hearing loss is one of the 10 most common causes of disability-adjusted life years worldwide [Olusanya, 2007]. The prevalence of disabling hearing loss is increasing, and in view of the demographic development and the increase in life expectancy, this might have a much greater impact than assumed in the future [Olusanya, 2007]. It is expected that by 2050 there will be $>900$ million people with disabling hearing loss [WHO, 2018]. Sensorineural hearing loss is mainly caused by loss or degeneration of auditory hair cells (HCs), spiral ganglion neurons, and synaptic functions. Damage to these structures is caused by noise exposure, age, genetics, infections, and exposure to drugs like aminoglycoside antibiotics and chemotherapeutic agents. Loss of the sensory cells of the inner ear, the auditory HCs, is a main cause of sensorineural hearing loss. In addition, the loss of HCs in mammals is irreversible as no regeneration occurs. While today we are able to effectively treat conductive hearing loss, this is not the case with sensorineural hearing loss as we do not yet have any medicines specifically approved to treat hearing loss; our current therapeutic options are limited to hearing aids and cochlear implants [Wang and Puel, 2018]. To find preventive and therapeutic strategies in the future

Daniel Bodmer

Clinic for Otolaryngology, Head and Neck Surgery

University of Basel Hospital, Petersgraben 4

CH-4031 Basel (Switzerland)

daniel.bodmer@usb.ch 
we depend on understanding molecular mechanisms involved in hearing loss.

Telmisartan is an angiotensin II receptor blocker mainly used to treat hypertension. Angiotensin II is the major hormone in the renin-angiotensin-aldosterone system regulating blood pressure and fluid volume [Higuchi et al., 2007]. Effects of angiotensin II are mediated through the angiotensin II type 1 receptor (AT1R) and the angiotensin II type 2 receptor (AT2R). Both receptors belong to the 7-transmembrane $\mathrm{G}$ protein-coupled receptor family [Higuchi et al., 2007]. While most of the physiological functions of angiotensin II are imparted by the AT1R, the role of the AT2R remains less known [Higuchi et al., 2007]. Telmisartan is highly selective for the AT1R and an insurmountable antagonist at this receptor [Miura et al., 2011]. The AT1R activates several heterotrimeric $G$ proteins, such as $G_{i} / G_{0}, G_{q / 11}, G_{12}$, and $G_{13}$, and thus activates phospholipases $\mathrm{A}_{2}, \mathrm{C}$, and $\mathrm{D}$, the nuclear factor- $\kappa \mathrm{B}(\mathrm{NF}-\kappa \mathrm{B})$ pathway, and different intracellular kinases [Higuchi et al., 2007; Hunyady and Catt, 2006]. Among the activated intracellular kinases are receptor and nonreceptor tyrosine kinases, serine/threonine kinases, mitogen-activated-signal-regulated kinases (MAPK), the Akt/protein kinase B, protein kinase $\mathrm{C}$, and p70-kDa ribosomal protein S6 kinase [Hunyady and Catt, 2006; Higuchi et al., 2007]. In addition, activation of AT1R by angiotensin II leads to the formation of reactive oxygen species (ROS) and activation of small GTPases, such as Ras, Rho, and Rac [Hunyady and Catt, 2006; Higuchi et al., 2007]. The AT1R also signals via G protein-independent pathways, like $\beta$-arrestin-mediated extracellular-signal-regulated kinase activation, and the Janus kinase/signal transducers and activators of transcription (Jak/STAT) pathway [Hunyady and Catt, 2006].

Besides being an AT1R antagonist, telmisartan was shown to be a partial agonist of the peroxisome proliferator-activated receptor $\gamma$ (PPAR- $\gamma$ ) [Benson et al., 2004]. PPAR- $\gamma$ belongs to the group of ligand-regulated nuclear hormone receptors (PPARs) that regulate lipid and glucose homeostasis, differentiation of inflammatory cells, but also cell proliferation [Berger and Moller, 2002]. As heterodimers with the retinoid X receptor the PPARs activate gene transcription upon ligand binding [Berger and Moller, 2002; Barish et al., 2006]. Specifically, several PPAR- $\gamma$ agonists inhibit the activation of NF- $\kappa B$, MAPK, STAT, and downregulate the expression of cytotoxic and proinflammatory gene products [Berger and Moller, 2002; Collino et al., 2006; Bright et al., 2008; Hamblin et al., 2009]. Interestingly, the statin simvastatin activates
PPAR- $\gamma$ [Yano et al., 2007], and in a previous study we have shown that simvastatin protected auditory HCs from gentamicin-induced toxicity [Brand et al., 2011]. In our recent study, we showed that PPAR- $\gamma$ and PPAR- $\alpha$ proteins are expressed in the mouse organ of Corti (OC), and that they are present in auditory HCs [Sekulic-Jablanovic et al., 2017]. Moreover, we found that different PPAR- $\gamma$ and PPAR- $\alpha$ agonists are protective against gentamicin-induced HC death [Sekulic-Jablanovic et al., 2017].

Furthermore, telmisartan has protective effects in the central nervous system [Tsukuda et al., 2009; Garrido-Gil et al., 2012; Eslami et al., 2014; Wang et al., 2014], in the cardiovascular system [Arozal et al., 2010; Maejima et al., 2011; Hu et al., 2014], and protects against gastric ulceration [Morsy et al., 2009], renal injury [Kusunoki et al., 2012; Zou et al., 2014; Ali et al., 2016], as well as hepatotoxicity [Kelleni et al., 2016]. In all these tissues, telmisartan had antioxidant properties and reduced the generation of ROS. Moreover, anti-inflammatory effects were involved in telmisartan-mediated protection.

These results prompted us to investigate a possible protective effect of telmisartan on gentamicin-damaged auditory HCs and whether the PPAR- $\gamma$ antagonist GW9662 could block this effect.

\section{Materials and Methods}

\section{OC Dissection}

All animal procedures were carried out in Basel, Switzerland, according to an animal research protocol approved by the Animal Care Committee of the Canton of Basel, Switzerland. Animals were sacrificed prior to all tissue extractions.

Cochlear explants were dissected from Wistar rat pups at postnatal day 5 (Janvier Labs, Le Genest-Saint-Isle, France). After sacrifice by decapitation of the pups, the mandible was removed and the skulls were opened midsagittally, followed by removal of the brain. The temporal bones were harvested and transferred to sterile phosphate-buffered saline ( $\mathrm{pH} 7.4$ ) in sterile 60-mm Petri dishes. Removal of the extra tissue and hard cartilage allowed for exposure of the cochlear capsule, whose bony and cartilaginous layers were peeled off to reveal the membranous labyrinth. After removal of the stria vascularis, the OC was removed in its entirety from base to apex. The spiral ganglion neurons, spiral limbus, and tectorial membrane were stripped off, and the remaining cochlear tissue composed of auditory HCs, supporting cells, and part of the basilar membrane functioned as one sample.

\section{Tissue Culture}

Isolated cochlear explants were initially placed in cell culture media containing Dulbecco's modified Eagle's medium supplemented with $10 \%$ fetal bovine serum, $25 \mathrm{mM}$ HEPES buffer, and $30 \mathrm{U} / \mathrm{mL}$ penicillin (all chemicals from Sigma Aldrich Chemie $\mathrm{GmbH}$, Steinheim, Germany, unless indicated otherwise). Follow- 
Fig. 1. Experimental timeline representing addition and duration of different treatments. DMSO, dimethyl sulfoxide.

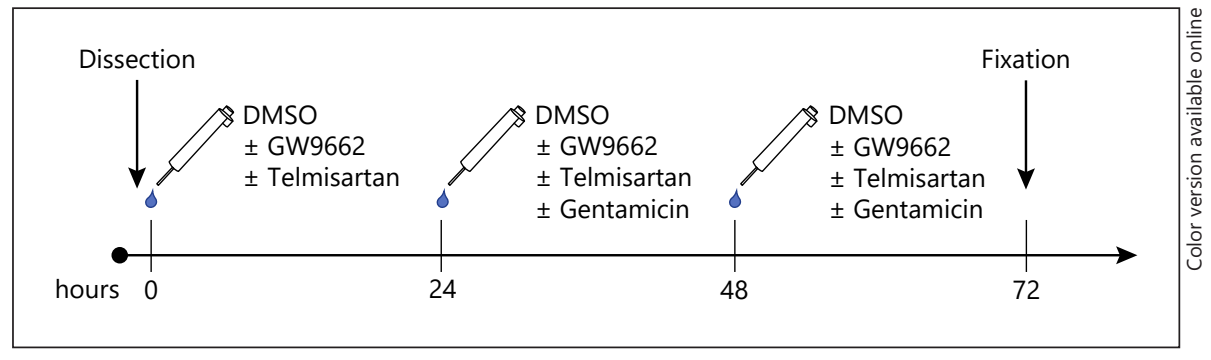

Table 1. The different treatment groups

\begin{tabular}{lllllll}
\hline Substance & \multicolumn{9}{l}{ Group } \\
\cline { 2 - 5 } & I & II & III & IV & V & VI \\
\hline $\begin{array}{l}\text { Control (dimethyl } \\
\quad \text { sulfoxide) }\end{array}$ & + & - & + & + & + & + \\
$\begin{array}{l}\text { Gentamicin (LD } \\
\text { Telmisartan }\end{array}$ & - & + & - & - & + & + \\
GW9662 & - & - & + & - & + & + \\
\hline
\end{tabular}

ing dissection, the culture medium was replaced with fresh cell culture medium and incubated at $37^{\circ} \mathrm{C}$ in $5 \% \mathrm{CO}_{2}$ for a 24 -h recovery period. For experiments with telmisartan (T8949) or with the PPAR- $\gamma$ antagonist GW9662 (M6191), a concentration of 10 $\mu \mathrm{M}$ telmisartan and/or $10 \mu \mathrm{M}$ GW9662, as well as an accompanying dimethyl sulfoxide (DMSO) control, were added to the recovery medium as a pretreatment (Fig. 1). Telmisartan and GW9662 media $(10 \mu \mathrm{M})$ had a final concentration of $0.1 \%$ DMSO; this concentration was also used in the control media and is known to cause no damage to HCs [Qi et al., 2008]. We selected the corresponding concentrations based on previous in vitro studies that found that $10 \mu \mathrm{M}$ of GW9662 blocked the effects of different concentrations of telmisartan $(10,20 \mu \mathrm{M})$ [Maejima et al., 2011; Kusunoki et al., 2012; Hu et al., 2014; Su et al., 2015].

Experiments with cochlear explants were done to (1) establish a median lethal dose $\left(\mathrm{LD}_{50}\right)$ of gentamicin (G1397), (2) test potential toxicity of telmisartan or GW9662 on HCs, (3) understand whether telmisartan affected gentamicin-induced damage to HCs, and (4) test whether GW9662 influenced telmisartan-mediated effects on gentamicin-induced damage to HCs. Following 24-h recovery or pretreatment, explants were exposed to drugs for $48 \mathrm{~h}$. Media were changed every $24 \mathrm{~h}$ to maintain concentration because of possible degradation after that period (Fig. 1). In order to find an $\mathrm{LD}_{50}$ of gentamicin, cochlear explants were exposed to gentamicin at concentrations of $30,60,90$, and $150 \mu \mathrm{M}$. The cochlear explants were exposed to gentamicin after the 24 -h recovery period, and again at hour 48, to mimic the way we would treat the explants with telmisartan and/or GW9662 in future experiments. Explants were fixed at hour 72 (Fig. 1). In the following experiments, explants were divided into the following groups (Table 1): (i) control (DMSO), (ii) gentamicin $\left(\mathrm{LD}_{50}\right)$, (iii) telmisartan, (iv) GW9662, (v) gentamicin $\left(\mathrm{LD}_{50}\right)$ and telmisartan, and (vi) gentamicin $\left(\mathrm{LD}_{50}\right)$, telmisartan, and GW9662.

Telmisartan Protects against GentamicinInduced HC Loss in vitro
Table 2. Numerical results of inner (IHC) and outer hair cell (OHC) numbers

\begin{tabular}{lrl}
\hline & IHC & OHC \\
\hline Apex & & \\
Control & $19.1 \pm 1.7$ & $58.8 \pm 3.2$ \\
$30 \mu \mathrm{M}$ gentamicin & $18.4 \pm 2.6$ & $43.9 \pm 13.7$ \\
$60 \mu \mathrm{M}$ gentamicin & $14.3 \pm 5.6$ & $42.8 \pm 14.2$ \\
$90 \mu \mathrm{M}$ gentamicin & $13.1 \pm 7.1$ & $36.5 \pm 13.2$ \\
$150 \mu \mathrm{M}$ gentamicin & $9.3 \pm 5.6$ & $36.6 \pm 19.7$ \\
Middle & & \\
Control & $19.3 \pm 1.4$ & $58.8 \pm 2.3$ \\
$30 \mu \mathrm{M}$ gentamicin & $18.1 \pm 3.2$ & $51.9 \pm 9.3$ \\
$60 \mu \mathrm{M}$ gentamicin & $13.8 \pm 6.1$ & $33.0 \pm 14.3$ \\
$90 \mu \mathrm{M}$ gentamicin & $13.3 \pm 4.9$ & $31.0 \pm 17.7$ \\
$150 \mu \mathrm{M}$ gentamicin & $7.0 \pm 5.9$ & $19.0 \pm 14.0$ \\
Base & & \\
Control & $19.2 \pm 1.3$ & $58.9 \pm 1.8$ \\
$30 \mu \mathrm{M}$ gentamicin & $17.5 \pm 4.7$ & $51.5 \pm 7.1$ \\
$60 \mu \mathrm{M}$ gentamicin & $13.4 \pm 5.8$ & $34.7 \pm 15.9$ \\
$90 \mu \mathrm{M}$ gentamicin & $11.7 \pm 6.2$ & $34.0 \pm 15.6$ \\
$150 \mu \mathrm{M}$ gentamicin & $8.4 \pm 5.8$ & $16.4 \pm 12.9$ \\
\hline
\end{tabular}

Values are shown as means \pm SDs. Cochlear explants were exposed to the indicated concentrations of gentamicin for 48 h. $n=$ 6-8 explants (3-4 animals) per condition. Data are expressed as the number of surviving hair cells per 20 IHCs, counted at different sites for the apical, middle, and basal turn of each explant.

\section{HC Visualization and Counts}

After treatment, the explants were fixed in $4 \%$ paraformaldehyde, permeabilized with Triton solution and stained with a 1:100 dilution of Alexa Fluor 568 phalloidin (Invitrogen AG, Basel, Switzerland). All images were taken with the Nikon A1R laser confocal microscope (Nikon AG Instruments, Egg, Switzerland). The surviving HCs were counted in sections corresponding to 20 inner hair cells (IHCs) at different sites of the basal, middle, and apical turns of each cochlear explant in randomly selected fields. Both IHCs and outer hair cells (OHCs) were counted to quantify $\mathrm{HC}$ survival. It was considered that the HCs had undergone apoptosis if there was a gap in the normal ordered array of HCs. The results are presented as the number of surviving HCs per cochlear turn. 

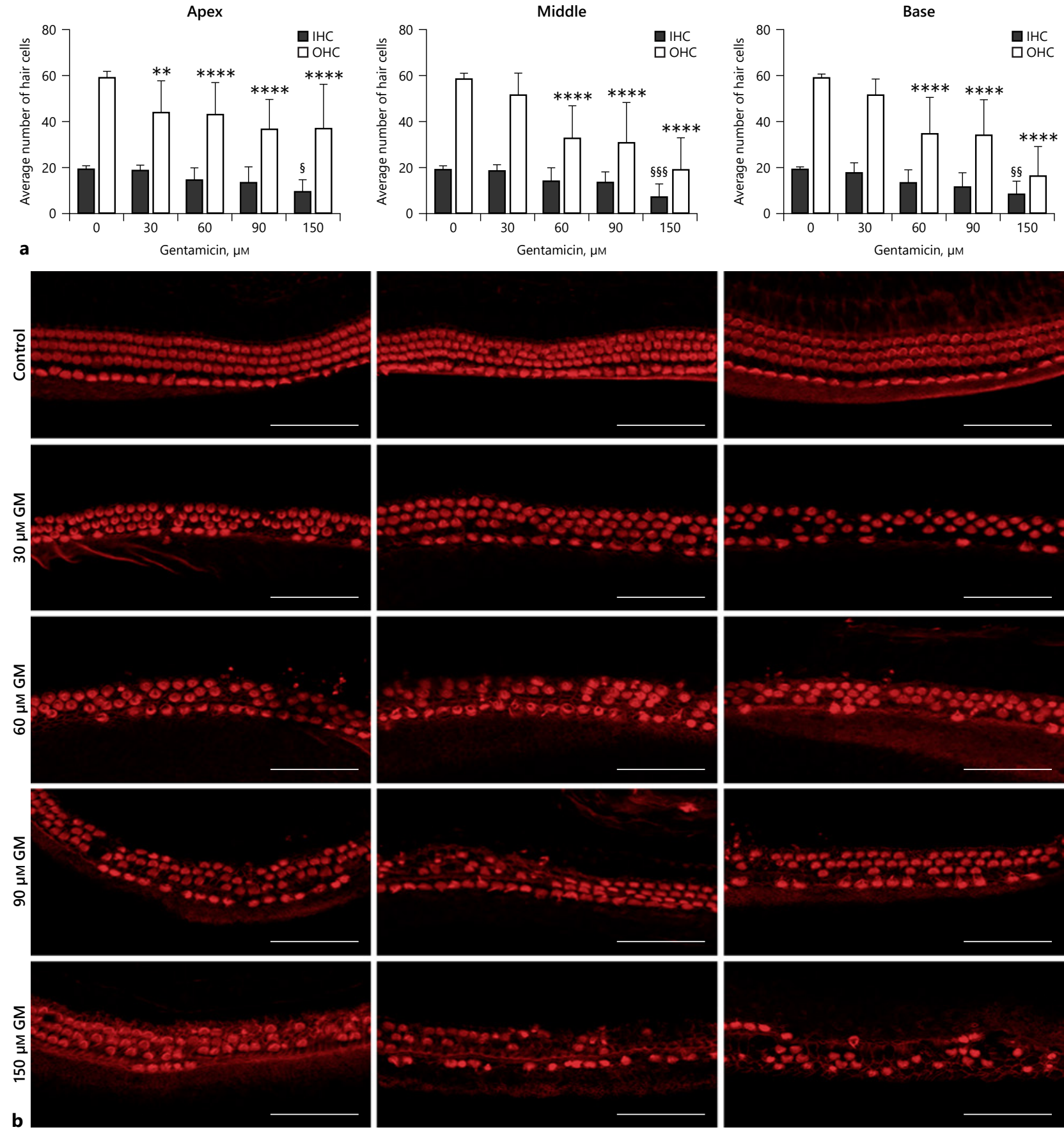

Fig. 2. Gentamicin ototoxicity in vitro. a Quantification of inner hair cells (IHCs) and outer hair cells (OHCs) exposed to the indicated concentrations of gentamicin (GM) for 48 h. b Representative images of phalloidin-stained hair cells (HCs). Gentamicin induced HC damage concentration dependently. $n=6-8$ explants per condition. Scale bar for all figures, $50 \mu \mathrm{M}$. Data are expressed as the number of surviving HCs per 20 IHCs, counted at different sites for the apical, middle, and basal turn of each explant. Values are shown as means + SDs. ${ }^{* *} p<0.01$ and ${ }^{* * * *} p<0.0001$ versus OHC control; ${ }^{\S} p<0.05,{ }^{\S \S} p<0.01$, and ${ }^{\S \S} p<0.001$ versus IHC control. 
Statistical Analysis

All data are presented as means \pm SDs. The statistical analysis was performed using Prism 7 (GraphPad software, La Jolla, CA, USA). We used two-way analysis of variance and Tukey's multiple comparisons test to analyze HC damage. The results were considered statistically significant when the $p$ value was $<0.05$.

\section{Results}

\section{Gentamicin-Induced HC Loss in the OC}

At a concentration of $30 \mu \mathrm{M}, \mathrm{OHC}$ counts were only significantly reduced in the apical turn $(43.9 \pm 13.7$, mean $\pm \mathrm{SD}, p<0.01)$ compared to untreated OHCs (58.8 \pm 3.2 ; Fig. 2a; Table 2). At a concentration of $60 \mu \mathrm{M}$, the number of surviving $\mathrm{OHCs}$ was significantly lower in all cochlear turns (apex $42.8 \pm 14.2$, middle $33.0 \pm 14.3$, base $34.7 \pm$ $15.9, p<0.0001)$ than the number of surviving OHCs in untreated explants (apex $58.8 \pm 3.2$, middle $58.8 \pm 2.3$, base $58.9 \pm 1.8$ ) and reached about $50 \%$ in the basal and middle turn (Fig. 2a; Table 2). Similar results were obtained when the explants were exposed to a slightly higher gentamicin concentration of $90 \mu \mathrm{M}$. At this concentration, there were significantly fewer surviving OHCs in all cochlear turns (apex $36.5 \pm 13.2$, middle $31.0 \pm 17.7$, base $34.0 \pm 15.6, p<0.0001)$ when compared to the number of surviving $\mathrm{OHCs}$ in untreated explants (Fig. 2a; Table 2). At a gentamicin concentration of $150 \mu \mathrm{M}$, there were significantly fewer surviving $\mathrm{OHCs}$ in all cochlear turns (apex $36.6 \pm 19.7$, middle $19.0 \pm 14.0$, base $16.4 \pm 12.9$, $p<0.0001)$ versus those in untreated cochlear explants (Fig. 2a; Table 2). At this concentration, much more OHCs underwent apoptosis and left a gap in the ordered rows of HCs (Fig. 2b). IHC counts were only significantly reduced at a gentamicin concentration of $150 \mu \mathrm{M}$ in all cochlear turns (apex 9.3 $\pm 5.6, p<0.05$; middle $7.0 \pm 5.9$, $p<0.001$; base $8.4 \pm 5.8, p<0.01$ ) when compared to the number of surviving IHCs in untreated explants (apex $19.1 \pm 1.7$, middle $19.3 \pm 1.4$, base $19.2 \pm 1.3$; Fig. 2 a; Table 2). The gentamicin concentration of $60 \mu \mathrm{M}$ was used in later experiments.

\section{Telmisartan and GW9662 Are Not Toxic to HCs in vitro}

IHC and OHC counts between a $0.1 \%$ DMSO vehicle control (OHCs, apex $59.9 \pm 0.3$, middle $59.6 \pm 1.2$, base $59.6 \pm 1.3$ ) and $10 \mu \mathrm{M}$ telmisartan (OHCs, apex $58.9 \pm 2.2$, middle $59.8 \pm 0.6$, base $59.3 \pm 1.5$ ) or $10 \mu \mathrm{M}$ GW9662 (OHCs, apex $59.1 \pm 2.6$, middle $58.7 \pm 1.8$, base $58.7 \pm 1.9$ ) did not differ significantly in all cochlear turns (Fig. 3a; Table 3). Thus, there is no HC damage induced by telmis-
Table 3. Numerical results of inner (IHC) and outer hair cell (OHC) numbers

\begin{tabular}{lll}
\hline & IHC & OHC \\
\hline Apex & & \\
$0.1 \%$ DMSO control & $18.5 \pm 2.3$ & $59.9 \pm 0.3$ \\
$10 \mu \mathrm{M}$ telmisartan & $17.9 \pm 3.5$ & $58.9 \pm 2.2$ \\
$10 \mu \mathrm{M}$ GW9662 & $18.9 \pm 1.5$ & $59.1 \pm 2.6$ \\
Middle & & \\
$0.1 \%$ DMSO control & $19.2 \pm 1.9$ & $59.6 \pm 1.2$ \\
$10 \mu \mathrm{M}$ telmisartan & $19.1 \pm 2.0$ & $59.8 \pm 0.6$ \\
$10 \mu \mathrm{M}$ GW9662 & $19.1 \pm 1.5$ & $58.7 \pm 1.8$ \\
Base & & \\
$0.1 \%$ DMSO control & $19.1 \pm 1.9$ & $59.6 \pm 1.3$ \\
$10 \mu \mathrm{M}$ telmisartan & $19.5 \pm 1.2$ & $59.3 \pm 1.5$ \\
$10 \mu \mathrm{M}$ GW9662 & $18.3 \pm 2.8$ & $58.7 \pm 1.9$ \\
\hline
\end{tabular}

Values are shown as means \pm SDs. DMSO, dimethyl sulfoxide. Cochlear explants were exposed to the indicated concentrations of telmisartan and GW9662 for 72 h. $n=6$ explants ( 3 animals) per condition. Data are expressed as the number of surviving hair cells per 20 IHCs, counted at different sites for the apical, middle, and basal turn of each explant.

artan or GW9662 alone. The undamaged HCs are seen as 3 intact rows of OHCs and one intact row of IHCs in cochlear explants exposed to telmisartan or GW9662 alone similar to the control group (Fig. 3b).

Telmisartan Protects OHCs from Gentamicin-Induced Toxicity in vitro, and GW9662 Inhibits this Protective Effect

As expected, exposure to gentamicin resulted in significant $\mathrm{OHC}$ loss in all cochlear turns (Fig. 4a). Thus, after exposure for $48 \mathrm{~h}$ to $60 \mu \mathrm{M}$ gentamicin, there were fewer surviving OHCs in a basal to apical gradient (apex $31.7 \pm 15.6$, middle $29.7 \pm 14.4$, base $26.1 \pm 10.9, p<$ 0.0001 ) compared to untreated OHCs (apex $58.3 \pm 2.5$, middle $59.4 \pm 1.2$, base $59.5 \pm 1.1$; Fig. 4 a; Table 4 ). Interestingly, in explants exposed to gentamicin and telmisar$\tan$, there were significantly more surviving OHCs in all cochlear turns (apex $47.7 \pm 12.0$, middle $43.6 \pm 14.5$, base $46.1 \pm 15.9, p<0.0001)$ than in explants exposed to gentamicin alone (Fig. 4a; Table 4). In cochlear explants exposed to gentamicin and telmisartan most of the OHCs survived since the explants showed only a few missing HCs (Fig. 4b). Despite protection against gentamicin damage, OHC counts in explants exposed to gentamicin and telmisartan were still significantly lower when compared to those in untreated cochlear explants (apex $p<$ 0.001 , middle and base $p<0.0001$; Fig. 4a). 


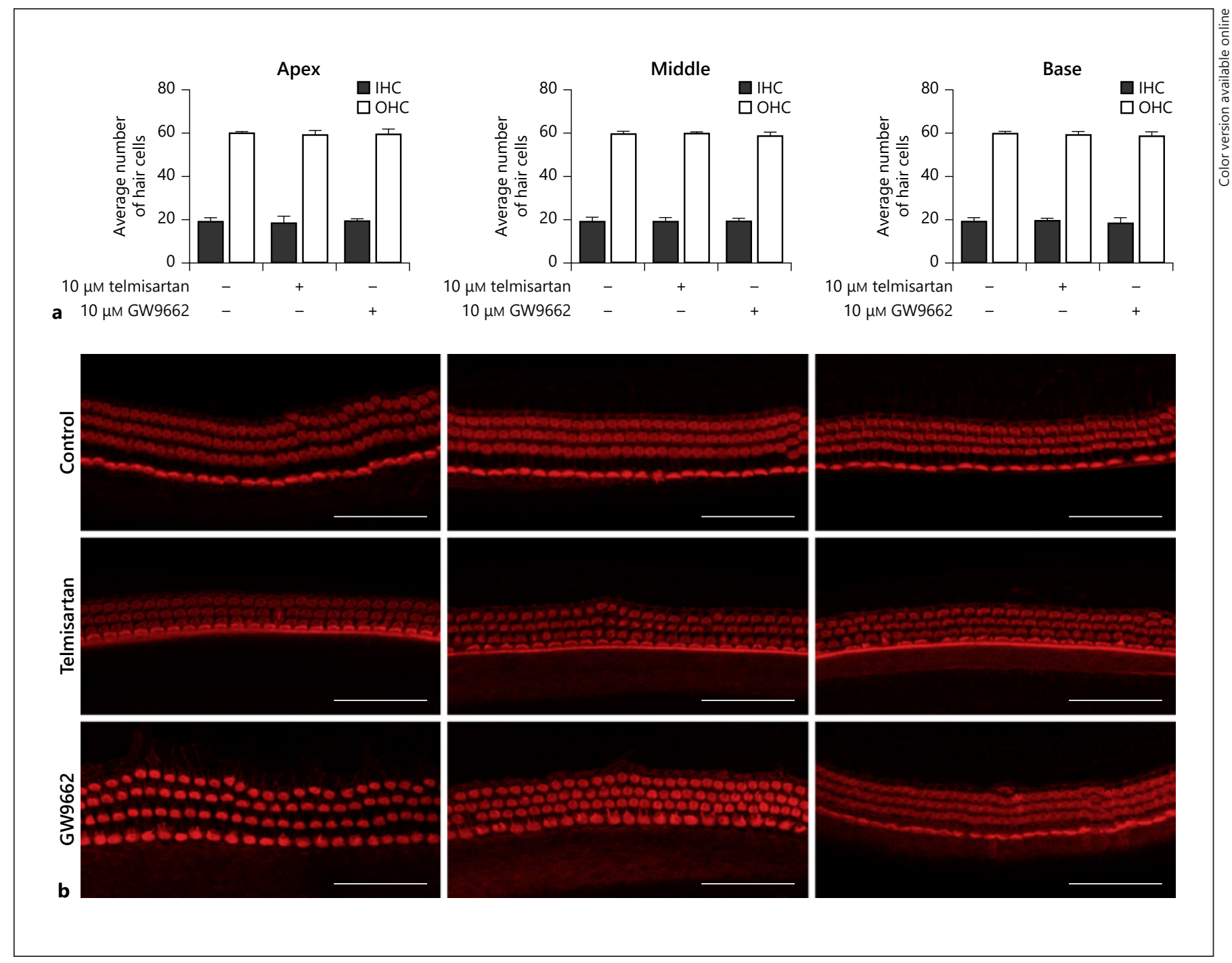

Fig. 3. Telmisartan and GW9662 are not toxic to hair cells (HCs) in vitro. a Quantification of inner hair cells (IHCs) and outer hair cells (OHCs) in cochlear explants exposed to the indicated concentrations of telmisartan and GW9662. b Representative images of phalloidin-stained HCs. Exposure to $10 \mu \mathrm{M}$ telmisartan or GW9662 for $72 \mathrm{~h}$ did not cause any HC loss when compared to a
$0.1 \%$ dimethyl sulfoxide control. $n=6$ explants per condition. Scale bar for all figures, $50 \mu \mathrm{M}$. Data are expressed as the number of surviving HCs per $20 \mathrm{IHCs}$, counted at different sites for the apical, middle, and basal turn of each explant. Values are shown as means + SDs.
In explants exposed to gentamicin, telmisartan, and GW9662, there were significantly fewer surviving OHCs in all cochlear turns (apex $22.7 \pm 17.5$, middle $21.3 \pm 15.3$, base $24.3 \pm 20.0$; $p<0.0001$ ) versus those exposed to gentamicin and telmisartan (apex $47.7 \pm 12.0$, middle $43.6 \pm$ 14.5 , base $46.1 \pm 15.9$; Fig. 4a; Table 4). In the basal turn, OHC counts in explants exposed to gentamicin, telmisartan, and GW9662 were similar to those in cochlear explants exposed to gentamicin alone. In contrast, in the apical and medial cochlear turn $\mathrm{OHC}$ counts were also significantly lower when compared to OHC counts in explants exposed to gentamicin alone $(p<0.01)$. Overall, explants exposed to all 3 drugs showed significantly reduced OHC counts compared to untreated explants in all cochlear turns $(p<0.0001)$. In those explants exposed to gentamicin, telmisartan, and GW9662, many OHCs were missing and left a gap in the ordered rows of HCs (Fig. 4b). In summary, GW9662 blocked the telmisartan-mediated protection on $\mathrm{OHCs}$. 

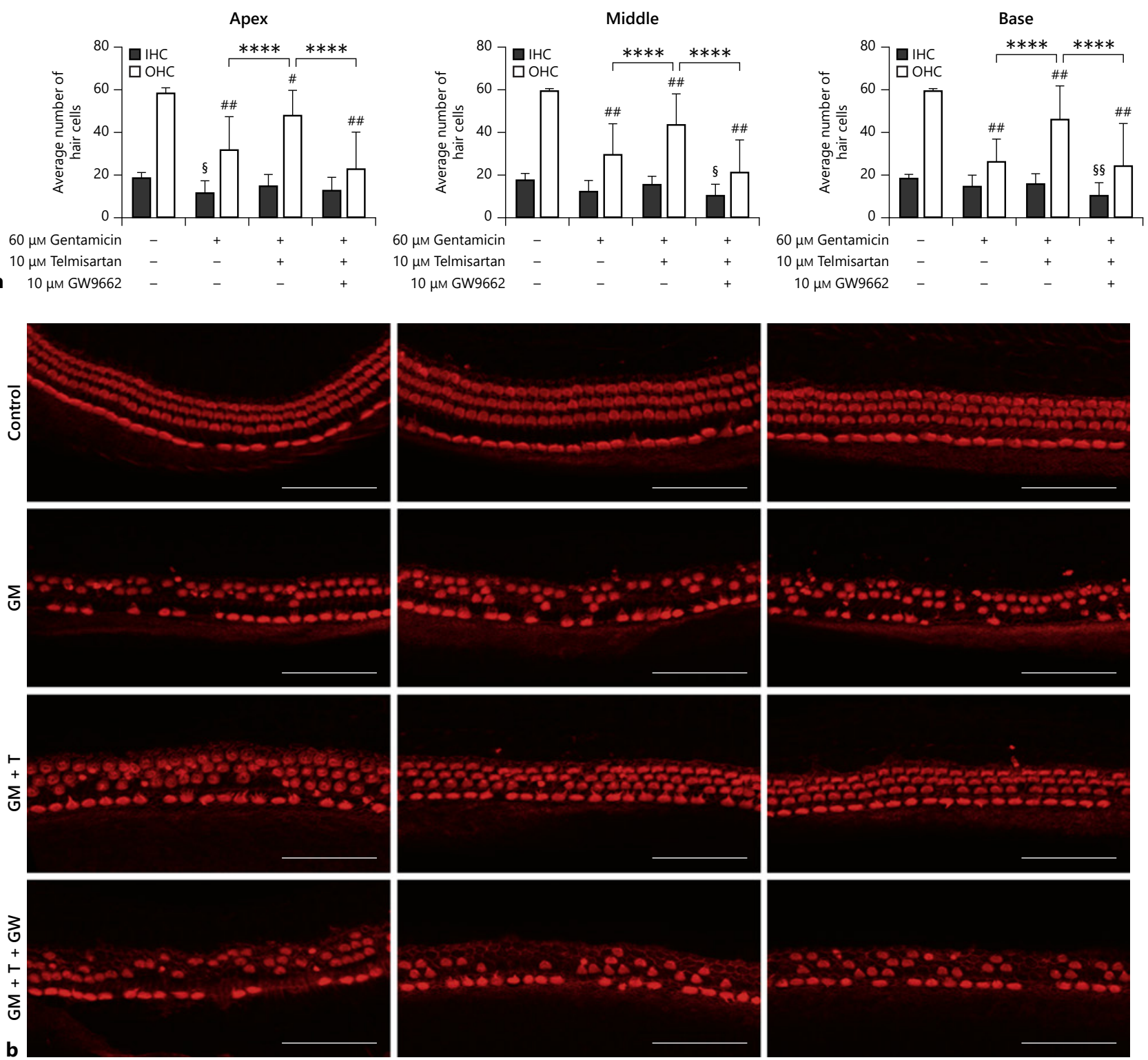

Fig. 4. Telmisartan protects outer hair cells (OHCs) from gentamicin-induced toxicity in vitro and GW9662 inhibits this protective effect. a Quantification of inner hair cells (IHCs) and OHCs in cochlear explants exposed to the indicated concentrations of gentamicin, telmisartan, and/or GW9662. b Representative images of phalloidin-stained hair cells (HCs). Exposure to $10 \mu \mathrm{M}$ telmisartan (T) in addition to $60 \mu \mathrm{M}$ gentamicin (GM) decreased OHC loss compared with exposure to $60 \mu \mathrm{M}$ gentamicin alone. Addition of $10 \mu \mathrm{M}$ GW9662 (GW) to gentamicin and telmisartan inhibited this protective effect as explants showed increased OHC loss, with more gaps in the normal ordered array of HCs. $n=10$ explants per condition. Scale bar for all figures, $50 \mu \mathrm{M}$. Data are expressed as the number of surviving HCs per 20 IHCs, counted at different sites for the apical, middle, and basal turn of each explant. Values are shown as means + SDs. ${ }^{* * * *} p<0.0001$ between 2 treated groups; ${ }^{\#} p<0.001$ and ${ }^{\# \#} p<0.0001$ versus OHC control ( $0.1 \%$ dimethyl sulfoxide); ${ }^{\S} p<0.05$ and ${ }^{\S} p<0.01$ versus IHC control $(0.1 \%$ dimethyl sulfoxide). 
Table 4. Numerical results of inner (IHC) and outer hair cell $(\mathrm{OHC})$ numbers

\begin{tabular}{|c|c|c|}
\hline & IHC & $\mathrm{OHC}$ \\
\hline \multicolumn{3}{|l|}{ Apex } \\
\hline $0.1 \%$ DMSO control & $18.2 \pm 3.0$ & $58.3 \pm 2.5$ \\
\hline $60 \mu \mathrm{M}$ gentamicin & $11.4 \pm 6.1$ & $31.7 \pm 15.6$ \\
\hline $10 \mu \mathrm{M}$ telmisartan $+60 \mu \mathrm{M}$ gentamicin & $14.7 \pm 5.7$ & $47.7 \pm 12.0$ \\
\hline $10 \mu \mathrm{M}$ GW9662 $+10 \mu \mathrm{M}$ telmisartan $+60 \mu \mathrm{M}$ gentamicin & $12.5 \pm 6.6$ & $22.7 \pm 17.5$ \\
\hline \multicolumn{3}{|l|}{ Middle } \\
\hline $0.1 \%$ DMSO control & $17.4 \pm 3.5$ & $59.4 \pm 1.2$ \\
\hline $60 \mu \mathrm{M}$ gentamicin & $11.9 \pm 5.4$ & $29.7 \pm 14.4$ \\
\hline $10 \mu \mathrm{M}$ telmisartan $+60 \mu \mathrm{M}$ gentamicin & $15.2 \pm 4.2$ & $43.6 \pm 14.5$ \\
\hline $10 \mu \mathrm{M}$ GW9662 $+10 \mu \mathrm{M}$ telmisartan $+60 \mu \mathrm{M}$ gentamicin & $10.1 \pm 5.7$ & $21.3 \pm 15.3$ \\
\hline \multicolumn{3}{|l|}{ Base } \\
\hline $0.1 \%$ DMSO control & $18.2 \pm 2.2$ & $59.5 \pm 1.1$ \\
\hline $60 \mu \mathrm{M}$ gentamicin & $14.7 \pm 5.4$ & $26.1 \pm 10.9$ \\
\hline $10 \mu \mathrm{M}$ telmisartan $+60 \mu \mathrm{M}$ gentamicin & $15.9 \pm 4.8$ & $46.1 \pm 15.9$ \\
\hline $10 \mu \mathrm{M}$ GW $9662+10 \mu \mathrm{M}$ telmisartan $+60 \mu \mathrm{M}$ gentamicin & $10.3 \pm 6.3$ & $24.3 \pm 20.0$ \\
\hline \multicolumn{3}{|c|}{$\begin{array}{l}\text { Values are shown as means } \pm \text { SDs. DMSO, dimethyl sulfoxide. Cochlear explants wer } \\
\text { exposed to the indicated concentrations of gentamicin, telmisartan, and/or GW9662. } n \\
10 \text { explants ( } 5 \text { animals) per condition. Data are expressed as the number of surviving hai } \\
\text { cells per } 20 \text { IHCs, counted at different sites for the apical, middle, and basal turn of eac } \\
\text { explant. }\end{array}$} \\
\hline
\end{tabular}

IHC counts were significantly reduced in gentamicin-, telmisartan-, and GW9662-treated explants in the basal and medial cochlear turns (middle $10.1 \pm 5.7, p<0.05$; base $10.3 \pm 6.3, p<0.01$ ), and in cochlear explants exposed to gentamicin alone in the apical turn (11.4 \pm 6 , $p<0.05)$ when compared to untreated cochlear explants (apex 18.2 \pm 3.0 , middle $17.4 \pm 3.5$, base $18.2 \pm 2.2$; Fig. 4a; Table 4).

\section{Discussion}

In the present work, we found that telmisartan protected HCs against gentamicin-induced toxicity in vitro. This protective effect was mediated by PPAR- $\gamma$. Telmisartan has pleiotropic effects and protects against damage in different cell types. Molecular events involved in telmisartan's protection include pathways that are involved in aminoglycoside ototoxicity. In a previous study we showed that PPAR- $\gamma$ agonists protect HCs from oxidative stress and death [Sekulic-Jablanovic et al., 2017] and the PPAR- $\gamma$ agonist pioglitazone was shown to protect against noiseinduced hearing loss [Paciello et al., 2018].

Gentamicin-Induced HC Loss in the OC

The aminoglycoside gentamicin is a known ototoxic agent, and various studies on HCs that used gentamicin as damaging agent have already shown different molecular mechanisms involved in HC survival and death [Battaglia et al., 2003; Chung et al., 2006; Brand et al., 2015].

\section{Telmisartan and GW9662 Are Not Toxic to HCs in vitro}

GW9662 is toxic to primary human peripheral blood mononuclear cell cultures [Jaudszus et al., 2014], oral squamous cell carcinoma cell lines [Masuda et al., 2005], and human colorectal adenocarcinoma cell lines [Schaefer et al., 2007]. It also potentiates neurotoxic effects in primary cultures of mouse cortical neurons, and this was discussed to be associated with non-PPAR- $\gamma$-specific effects of GW9662 [Wojtowicz et al., 2014]. Given these results and the protective role of PPAR- $\gamma$ agonists on $\mathrm{HCs}$ demonstrated in our earlier study [Sekulic-Jablanovic et al., 2017], we wanted to rule out that GW9662 is toxic to HCs.

As demonstrated in our study, GW9662 and telmisartan are not toxic to HCs at our concentration used. Our results are consistent with previous studies, where GW9662 and/or telmisartan showed no toxic effects [Zhao et al., 2006; Garrido-Gil et al., 2012; Eslami et al., 2014; Pang et al., 2014]. These results suggest that PPAR- $\gamma$ signaling is not essential for $\mathrm{HC}$ survival under normal conditions. 
Telmisartan Protects OHCs from Gentamicin-Induced Toxicity in vitro, and GW9662 Inhibits this Protective Effect

Telmisartan significantly diminished OHC loss in all cochlear turns in explants exposed to gentamicin plus telmisartan versus explants exposed to gentamicin only. There are no other studies in the literature that investigated telmisartan-mediated effects on auditory HCs. However, our findings are consistent with other studies that demonstrated neuroprotective effects of telmisar$\tan$ in various in vitro [Eslami et al., 2014; Pang et al., 2014; Wang et al., 2014] and in vivo [Jung et al., 2007; Mogi et al., 2008; Tsukuda et al., 2009; Thoene-Reineke et al., 2011; Garrido-Gil et al., 2012; Justin et al., 2014; Wincewicz et al., 2016] experimental models. Moreover, telmisartan also has protective effects on other organ systems such as the cardiovascular system [Arozal et al., 2010; Maejima et al., 2011; Hu et al., 2014], the stomach [Morsy et al., 2009], the kidneys [Kusunoki et al., 2012; Zou et al., 2014; Ali et al., 2016], and the liver [Kelleni et al., 2016].

GW9662, a potent, irreversible, and selective PPAR- $\gamma$ antagonist [Leesnitzer et al., 2002], completely inhibited telmisartan-mediated protection on HCs. These results suggest that telmisartan's protective effect on HCs is due to its agonistic activity on PPAR- $\gamma$. In previous studies, GW9662 also partially [Mogi et al., 2008; Eslami et al., 2014; Wang et al., 2014] or completely inhibited [Tsukuda et al., 2009; Garrido-Gil et al., 2012; Pang et al., 2014] telmisartan-mediated protective properties in other cellular systems than the inner ear, which is in line with our results. Expression of the AT1R or AT2R has not been investigated in the inner ear. However, of the class of angiotensin II receptor blockers, telmisartan has the strongest affinity for PPAR- $\gamma$ [Marshall et al., 2006], and in our recent study we demonstrated PPAR- $\gamma$ expression in cochlear explants from 5-day-old mice. We also localized PPAR- $\gamma$ expression in both IHCs and OHCs, in the stria vascularis, spiral ganglion, and cochlear nerve but also in supporting cells and cells of the basal lamina of adult mice [Sekulic-Jablanovic et al., 2017]. Since we used rats in the present study, we assume that PPAR- $\gamma$ is expressed similarly in the rat cochlea. Taken together, PPAR- $\gamma$ expression in auditory HCs and inhibition of telmisartan's protective effect by GW9662 suggest PPAR- $\gamma$ signaling as protective mechanism in HCs. These results are consistent with our previous study [Sekulic-Jablanovic et al., 2017] and with studies of other groups [Paciello et al., 2018; Park et al., 2017] that demonstrated protection from gentamicin-induced $\mathrm{HC}$ death and noise-induced hearing loss mediated by PPAR agonists. Ten micromolars of both the PPAR- $\gamma$-selective agonist pioglitazone or the PPAR- $\gamma / \alpha$ dual agonist tesaglitazar offered full protection against gentamicin-induced $\mathrm{HC}$ loss in vitro [Sekulic-Jablanovic et al., 2017], whereas $10 \mu \mathrm{M}$ telmisartan only offered partial protection in our study. This difference could be explained by partial PPAR- $\gamma$ agonism of telmisartan, whereas pioglitazone and tesaglitazar are full agonists.

Interestingly, in the apical and medial cochlear turns, OHC numbers in explants exposed to gentamicin, telmisartan, and GW9662 were also significantly lower when compared to OHC numbers in explants exposed to gentamicin alone. Moreover, IHC numbers were significantly reduced in gentamicin-, telmisartan-, and GW9662treated explants when compared to untreated cochlear explants in the middle and basal turns. As GW9662 was not toxic to HCs, this raises the questions whether blocking PPAR $-\gamma$ in the presence of gentamicin potentiates gentamicin-induced HC toxicity. Interestingly, GarridoGil et al. [2012] found similar results in that administration of GW9662 significantly increased neurotoxin 1-methyl-4-phenyl-1,2,3,6-tetrahydropyridine-induced dopaminergic neuron death in AT1R deficient mice, and it potentiated tetrabromobisphenol A neurotoxicity in primary neocortical cell cultures [Wojtowicz et al., 2014]. In contrast, this phenomenon has not been observed in other studies where GW9662 blocked telmisartan-mediated protective effects [Pang et al., 2014; Wang et al., 2014]. Therefore, possible effects of PPAR- $\gamma$ inhibition on toxic events in HCs need to be further investigated in the future.

In the present study we demonstrate that telmisartan protects auditory HCs from gentamicin-induced ototoxicity. From this the question arises, by which mechanisms telmisartan can exert this PPAR- $\gamma$ dependent protective effect. Since we did not investigate this in our study, we can only hypothesize about the underlying mechanisms. Telmisartan exerts protective effects in many cell types through PPAR- $\gamma$ signaling [Morsy et al., 2009; Maejima et al., 2011; Kusunoki et al., 2012; Eslami et al., 2014; Zou et al., 2014]. The nuclear receptor PPAR- $\gamma$ forms a heterodimer with the retinoid $\mathrm{X}$ receptor, and upon ligand binding it also binds to peroxisome proliferator response elements and induces gene transcription of many target genes [Berger and Moller, 2002; Fang et al., 2016]. After activation, PPAR- $\gamma$ signaling regulates among others NF$\kappa \mathrm{B}$, tumor necrosis factor- $\alpha$, and nuclear factor erythroid 2-related factor 2 signaling [Berger and Moller, 2002; Cai et al., 2018], all of which are also involved in hair cell 
death and survival [Nagy et al., 2005; Dinh et al., 2008; Hoshino et al., 2011].

Main molecular mechanisms underlying telmisartanmediated protection are anti-inflammatory [Jung et al., 2007; Tsukuda et al., 2009] and antioxidative effects [Arozal et al., 2010; Eslami et al., 2014; Jung et al., 2007; Morsy et al., 2009]. Telmisartan attenuates the expression of proinflammatory cytokines such as tumor necrosis factor- $\alpha$ [Thoene-Reineke et al., 2011; Tsukuda et al., 2009], interleukin-1 $\beta$, and interleukin-6 [Justin et al., 2014], decreases cyclooxygenase- 2 expression [Jung et al., 2007], and increases anti-inflammatory cytokine interleukin-10 levels [Justin et al., 2014]. Moreover, telmisartan diminishes intracellular ROS, malondialdehyde as well as NADPH oxidase levels, elevates glutathione levels as well as superoxide dismutase and catalase activity [Morsy et al., 2009; Arozal et al., 2010; Eslami et al., 2014; Kelleni et al., 2016], and ameliorates lipid peroxide levels [Justin et al., 2014]. In addition, telmisartan activates the phosphatidylinositol 3-kinase (PI3K)/Akt/glycogen synthase kinase-3 $\beta$ (GSK-3 $\beta$ ) pathway [Pang et al., 2014; Wang et al., 2014]. Aminoglycoside antibiotics are mainly toxic through generation of ROS [Wang and Puel, 2018]. They have also been shown to trigger inflammatory responses [Abi-Hachem et al., 2010] and to induce the production of proinflammatory cytokines in cochlear explants in vitro [Bas et al., 2012]. The PI3K/Akt pathway is a common mechanism promoting $\mathrm{HC}$ survival in the inner ear [Brand et al., 2015], and it has been shown to oppose gentamicin toxicity in HCs in vitro [Chung et al., 2006]. In addition, selective GSK-3 $\beta$ inhibitors diminish cisplatin-induced auditory $\mathrm{HC}$ death in vitro and protect against cisplatin-induced hearing loss in vivo [Park et al., 2009].

Interestingly, the PPAR- $\gamma$ agonist pioglitazone protects against noise-induced hearing loss through both anti-inflammatory effects and by reducing oxidative stress [Paciello et al., 2018]. Indeed, in our recent in vitro study using a gentamicin ototoxicity model we saw that pioglitazone was able to counteract the gentamicin-induced increase in ROS and lipid peroxidation levels, and to inhibit apoptosis by enhancing the cell's intrinsic antioxidant defense system [Sekulic-Jablanovic et al., 2017]. Besides PPAR- $\gamma$, other PPAR subtypes also possess protective properties in auditory HCs [Park et al., 2017; Sekulic-Jablanovic et al., 2017; Kim et al., 2018]. Fenofibrate, a PPAR- $\alpha$ agonist, protects auditory HCs against gentamicin- and cisplatin-induced ototoxicity reducing oxidative stress [Park et al., 2017; Kim et al., 2018]. Interestingly, Kim et al. [2018] also showed that fenofibrate pro- tects against mitochondrial and peroxisomal dysfunction by increasing PPAR- $\gamma$, PPAR- $\alpha$, and PPAR coactivator $1 \alpha$ expression and by increasing fatty acid-binding protein expression.

Taken together, these results suggest that anti-inflammatory and antioxidative effects as well as activation of the PI3K/Akt/GSK-3 $\beta$ pathway may also be involved in telmisartan-mediated protection in HCs. Moreover, other mechanisms such as protection against mitochondrial and peroxisomal dysfunction may also be involved. In addition, it is possible that the involvement of these mechanisms in HC protection may vary depending on the nature of the damage and the condition of the cells. Finally, the AT1R was shown to activate ROS production and other pathways involved in $\mathrm{HC}$ death, such as the NF- $\kappa \mathrm{B}$, c-Jun N-terminal kinase, and p38MAPK pathway, and to activate small GTPases, such as Ras, Rho, and Rac [Hunyady and Catt, 2006; Higuchi et al., 2007]. Although we showed that telmisartan's protection on HCs was mediated by PPAR- $\gamma$ signaling, antagonistic effects at the AT1R might also be involved. Future studies will need to identify the specific molecular mechanisms involved in telmisartan's mediated protection on HCs.

\section{Conclusion}

In our study we demonstrated for the first time that telmisartan protects auditory HCs against gentamicininduced damage in vitro. This protective effect is presumably mediated through PPAR- $\gamma$ signaling, which is expressed in auditory HCs [Sekulic-Jablanovic et al., 2017].

Telmisartan is highly lipophilic and has a high volume of distribution and therefore possesses high tissue-penetrating properties [Wienen et al., 2000]. Telmisartan has been shown to penetrate the blood-brain barrier [Gohlke et al., 2001] and revealed neuroprotective properties in models of Parkinson's disease [Garrido-Gil et al., 2012], stroke [Thoene-Reineke et al., 2011], glutamate-induced neurotoxicity [Wang et al., 2014], stress-induced cognitive decline [Wincewicz et al., 2016], and Alzheimer's disease [Mogi et al., 2008; Tsukuda et al., 2009]. Together with a long terminal elimination half-life in humans (approx. 22-24 h) [Wienen et al., 2000], it is an interesting drug candidate as it could cross the blood-labyrinth barrier.

To conclude, telmisartan is a promising agent that could be used in the future to address sensorineural hearing loss. 


\section{Acknowledgment}

The authors would like to acknowledge the Microscopy Core Facility of the Department of Biomedicine at the University of Basel for their technical assistance and the Animal Facility of the Department of Biomedicine for its support in animal care.

\section{Statement of Ethics}

All animal experiments were performed according to Swiss Animal Welfare laws and following an animal research protocol approved by the Animal Care Committee of the Canton of Basel, Switzerland (Kantonales Veterinäramt Basel, permission No.: 2263).

\section{Disclosure Statement}

The authors have no conflicts of interest to declare.

\section{Funding Sources} close.

The authors have no funding or financial relationships to dis-

\section{Author Contributions}

D.B.: conceptualization. M.C., E.W., and N.J.: acquisition, analysis. M.C., S.L., and D.B.: interpretation. D.B.: resources. D.B. and S.L.: supervision. M.C.: writing.

\section{References}

Abi-Hachem RN, Zine A, Van De Water TR. The injured cochlea as a target for inflammatory processes, initiation of cell death pathways and application of related otoprotectives strategies. Recent Patents CNS Drug Discov. 2010 Jun;5(2):147-63.

Ali SI, Alhusseini NF, Atteia HH, Idris RA, Hasan RA. Renoprotective effect of a combination of garlic and telmisartan against ischemia/reperfusion-induced kidney injury in obese rats. Free Radic Res. 2016 Sep;50(9):966-86.

Arozal W, Watanabe K, Veeraveedu PT, Thandavarayan RA, Harima M, Sukumaran V, et al. Effect of telmisartan in limiting the cardiotoxic effect of daunorubicin in rats. J Pharm Pharmacol. 2010 Dec;62(12):1776-83.

Barish GD, Narkar VA, Evans RM. PPAR delta: a dagger in the heart of the metabolic syndrome. J Clin Invest. 2006 Mar;116(3):590-7.

Bas E, Van De Water TR, Gupta C, Dinh J, Vu L, Martínez-Soriano F, et al. Efficacy of three drugs for protecting against gentamicin-induced hair cell and hearing losses. Br J Pharmacol. 2012 Jul;166(6):1888-904.

Battaglia A, Pak K, Brors D, Bodmer D, Frangos JA, Ryan AF. Involvement of ras activation in toxic hair cell damage of the mammalian cochlea. Neuroscience. 2003;122(4):1025-35.

Benson SC, Pershadsingh HA, Ho CI, Chittiboyina A, Desai P, Pravenec M, et al. Identification of telmisartan as a unique angiotensin II receptor antagonist with selective PPARgamma-modulating activity. Hypertension. 2004 May;43(5):993-1002.

Berger J, Moller DE. The mechanisms of action of PPARs. Annu Rev Med. 2002;53(1):409-35.

Brand Y, Levano S, Radojevic V, Naldi AM, Setz C, Ryan AF, et al. All Akt isoforms (Akt1, Akt2, Akt3) are involved in normal hearing, but only Akt 2 and Akt 3 are involved in auditory hair cell survival in the mammalian inner ear. PLoS One. 2015 Mar;10(3):e0121599.

Brand Y, Setz C, Levano S, Listyo A, Chavez E, Pak $\mathrm{K}$, et al. Simvastatin protects auditory hair cells from gentamicin-induced toxicity and activates Akt signaling in vitro. BMC Neurosci. 2011 Nov;12(1):114.

Bright JJ, Kanakasabai S, Chearwae W, Chakraborty S. PPAR Regulation of Inflammatory Signaling in CNS Diseases. PPAR Res. 2008; 2008:658520.

Cai W, Yang T, Liu H, Han L, Zhang K, Hu X, et al. Peroxisome proliferator-activated receptor $\gamma$ (PPAR $\gamma$ ): A master gatekeeper in CNS injury and repair. Prog Neurobiol. 2018 Apr - May;163-164:27-58.

Chung WH, Pak K, Lin B, Webster N, Ryan AF. A PI3K pathway mediates hair cell survival and opposes gentamicin toxicity in neonatal rat organ of Corti. J Assoc Res Otolaryngol. 2006 Dec;7(4):373-82.

Collino M, Aragno M, Mastrocola R, Gallicchio M, Rosa AC, Dianzani C, et al. Modulation of the oxidative stress and inflammatory response by PPAR-gamma agonists in the hippocampus of rats exposed to cerebral ischemia/reperfusion. Eur J Pharmacol. 2006 Jan; 530(1-2):70-80.

Dinh CT, Haake S, Chen S, Hoang K, Nong E, Eshraghi AA, et al. Dexamethasone protects organ of corti explants against tumor necrosis factor-alpha-induced loss of auditory hair cells and alters the expression levels of apoptosis-related genes. Neuroscience. 2008 Nov; 157(2):405-13.

Eslami H, Sharifi AM, Rahimi H, Rahati M. Protective effect of telmisartan against oxidative damage induced by high glucose in neuronal PC12 cell. Neurosci Lett. 2014 Jan;558:31-6.

Fang L, Zhang M, Li Y, Liu Y, Cui Q, Wang N. PPARgene: A Database of Experimentally Verified and Computationally Predicted PPAR Target Genes. PPAR Res. 2016;2016: 6042162.

Garrido-Gil P, Joglar B, Rodriguez-Perez AI, Guerra MJ, Labandeira-Garcia JL. Involvement of PPAR- $\gamma$ in the neuroprotective and anti-inflammatory effects of angiotensin type
1 receptor inhibition: effects of the receptor antagonist telmisartan and receptor deletion in a mouse MPTP model of Parkinson's disease. J Neuroinflammation. 2012 Feb;9(1):38.

Gohlke P, Weiss S, Jansen A, Wienen W, Stangier J, Rascher W, et al. AT1 receptor antagonist telmisartan administered peripherally inhibits central responses to angiotensin II in conscious rats. J Pharmacol Exp Ther. 2001 Jul; 298(1):62-70.

Hamblin M, Chang L, Fan Y, Zhang J, Chen YE. PPARs and the cardiovascular system. Antioxid Redox Signal. 2009 Jun;11(6):1415-52.

Higuchi S, Ohtsu H, Suzuki H, Shirai H, Frank GD, Eguchi S. Angiotensin II signal transduction through the AT1 receptor: novel insights into mechanisms and pathophysiology. Clin Sci (Lond). 2007 Apr;112(8):417-28.

Hoshino T, Tabuchi K, Nishimura B, Tanaka S, Nakayama M, Ishii T, et al. Protective role of Nrf2 in age-related hearing loss and gentamicin ototoxicity. Biochem Biophys Res Commun. 2011 Nov;415(1):94-8.

Hu ZP, Fang XL, Qian HY, Fang N, Wang BN, Wang Y. Telmisartan prevents angiotensin IIinduced endothelial dysfunction in rabbit aorta via activating $\mathrm{HGF} / \mathrm{Met}$ system and PPAR $\gamma$ pathway. Fundam Clin Pharmacol. 2014 Oct;28(5):501-11.

Hunyady L, Catt KJ. Pleiotropic AT1 receptor signaling pathways mediating physiological and pathogenic actions of angiotensin II. Mol Endocrinol. 2006 May;20(5):953-70.

Jaudszus A, Lorkowski S, Gruen M, Roth A, Jahreis G. Limited Applicability of GW9662 to Elucidate PPAR $\gamma$-Mediated Fatty Acid Effects in Primary Human T-Helper Cells. Int J Inflamm. 2014;2014:149628.

Jung KH, Chu K, Lee ST, Kim SJ, Song EC, Kim $\mathrm{EH}$, et al. Blockade of AT1 receptor reduces apoptosis, inflammation, and oxidative stress in normotensive rats with intracerebral hemorrhage. J Pharmacol Exp Ther. 2007 Sep; 322(3):1051-8. 
Justin A, Sathishkumar M, Sudheer A, Shanthakumari S, Ramanathan M. Non-hypotensive dose of telmisartan and nimodipine produced synergistic neuroprotective effect in cerebral ischemic model by attenuating brain cytokine levels. Pharmacol Biochem Behav. 2014 Jul; 122:61-73.

Kelleni MT, Ibrahim SA, Abdelrahman AM. Effect of captopril and telmisartan on methotrexate-induced hepatotoxicity in rats: impact of oxidative stress, inflammation and apoptosis. Toxicol Mech Methods. 2016 Jun;26(5): 371-7.

Kim SJ, Park C, Lee JN, Park R. Protective roles of fenofibrate against cisplatin-induced ototoxicity by the rescue of peroxisomal and mitochondrial dysfunction. Toxicol Appl Pharmacol. 2018 Aug;353:43-54.

Kusunoki H, Taniyama Y, Azuma J, Iekushi K, Sanada F, Otsu R, et al. Telmisartan exerts renoprotective actions via peroxisome proliferator-activated receptor- $\gamma /$ hepatocyte growth factor pathway independent of angiotensin II type 1 receptor blockade. Hypertension. 2012 Feb;59(2):308-16.

Leesnitzer LM, Parks DJ, Bledsoe RK, Cobb JE, Collins JL, Consler TG, et al. Functional consequences of cysteine modification in the ligand binding sites of peroxisome proliferator activated receptors by GW9662. Biochemistry. 2002 May;41(21):6640-50.

Maejima Y, Okada H, Haraguchi G, Onai Y, Kosuge $H$, Suzuki J, et al. Telmisartan, a unique $A R B$, improves left ventricular remodeling of infarcted heart by activating PPAR gamma. Lab Invest. 2011 Jun;91(6):932-44.

Marshall TG, Lee RE, Marshall FE. Common angiotensin receptor blockers may directly modulate the immune system via VDR, PPAR and CCR2b. Theor Biol Med Model. 2006 Jan; $3(1): 1$.

Masuda T, Wada K, Nakajima A, Okura M, Kudo C, Kadowaki T, et al. Critical role of peroxisome proliferator-activated receptor gamma on anoikis and invasion of squamous cell carcinoma. Clin Cancer Res. 2005 Jun;11(11): 4012-21.

Miura S, Karnik SS, Saku K. Review: angiotensin II type 1 receptor blockers: class effects versus molecular effects. J Renin Angiotensin Aldosterone Syst. 2011 Mar;12(1):1-7.

Mogi M, Li JM, Tsukuda K, Iwanami J, Min LJ, Sakata A, et al. Telmisartan prevented cognitive decline partly due to PPAR-gamma activation. Biochem Biophys Res Commun. 2008 Oct;375(3):446-9.
Morsy M, Ashour O, Amin E, Rofaeil R. Gastroprotective effects of telmisartan on experimentally-induced gastric ulcers in rats. Pharmazie. 2009 Sep;64(9):590-4.

Nagy I, Monge A, Albinger-Hegyi A, Schmid S, Bodmer D. NF-kappaB is required for survival of immature auditory hair cells in vitro. J Assoc Res Otolaryngol. 2005 Sep;6(3):260-8.

Olusanya BO. "The right stuff”: the global burden of disease. PLoS Med. 2007 Feb;4(2):e84.

Paciello F, Fetoni AR, Rolesi R, Wright MB, Grassi C, Troiani D, et al. Pioglitazone Represents an Effective Therapeutic Target in Preventing Oxidative/Inflammatory Cochlear Damage Induced by Noise Exposure. Front Pharmacol. 2018 Oct;9:1103.

Pang T, Sun LX, Wang T, Jiang ZZ, Liao H, Zhang LY. Telmisartan protects central neurons against nutrient deprivation-induced apoptosis in vitro through activation of PPAR $\gamma$ and the Akt/GSK-3 $\beta$ pathway. Acta Pharmacol Sin. 2014 Jun;35(6):727-37.

Park C, Ji HM, Kim SJ, Kil SH, Lee JN, Kwak S, et al. Fenofibrate exerts protective effects against gentamicin-induced toxicity in cochlear hair cells by activating antioxidant enzymes. Int J Mol Med. 2017 Apr;39(4):960-8.

Park HJ, Kim HJ, Bae GS, Seo SW, Kim DY, Jung WS, et al. Selective GSK-3beta inhibitors attenuate the cisplatin-induced cytotoxicity of auditory cells. Hear Res. 2009 Nov;257(1-2): $53-62$.

Qi W, Ding D, Salvi RJ. Cytotoxic effects of dimethyl sulphoxide (DMSO) on cochlear organotypic cultures. Hear Res. 2008 Feb;236(12):52-60.

Schaefer KL, Takahashi H, Morales VM, Harris G, Barton S, Osawa E, et al. PPARgamma inhibitors reduce tubulin protein levels by a PPARgamma, PPARdelta and proteasomeindependent mechanism, resulting in cell cycle arrest, apoptosis and reduced metastasis of colorectal carcinoma cells. Int J Cancer. 2007 Feb;120(3):702-13.

Sekulic-Jablanovic M, Petkovic V, Wright MB, Kucharava K, Huerzeler N, Levano S, et al. Effects of peroxisome proliferator activated receptors (PPAR)- $\gamma$ and $-\alpha$ agonists on cochlear protection from oxidative stress. PLoS One. 2017 Nov;12(11):e0188596.

Su X, Yu R, Yang X, Zhou G, Wang Y, Li L, et al. Telmisartan attenuates peritoneal fibrosis via peroxisome proliferator-activated receptor- $\gamma$ activation in rats. Clin Exp Pharmacol Physiol. 2015 Jun;42(6):671-9.

Thoene-Reineke C, Rumschüssel K, Schmerbach K, Krikov M, Wengenmayer C, Godes M, et al. Prevention and intervention studies with telmisartan, ramipril and their combination in different rat stroke models. PLoS One. 2011;6(8):e23646.
Tsukuda K, Mogi M, Iwanami J, Min LJ, Sakata A, Jing F, et al. Cognitive deficit in amyloid-betainjected mice was improved by pretreatment with a low dose of telmisartan partly because of peroxisome proliferator-activated receptor-gamma activation. Hypertension. 2009 Oct;54(4):782-7.

Wang J, Pang T, Hafko R, Benicky J, Sanchez-Lemus E, Saavedra JM. Telmisartan ameliorates glutamate-induced neurotoxicity: roles of AT(1) receptor blockade and PPAR $\gamma$ activation. Neuropharmacology. 2014 Apr;79:24961.

Wang J, Puel JL. Toward Cochlear Therapies. Physiol Rev. 2018 Oct;98(4):2477-522.

WHO. WHO Global estimates on prevalence of hearing loss. Available from: http://www. who.int/pbd/deafness/estimates/en/, 2018.

Wienen W, Entzeroth M, van Meel JC, Stangier J, Busch U, Ebner T, et al. A review on telmisartan: A novel, long-acting angiotensin II-receptor antagonist. Cardiovasc Drug Rev. 2000;18(2):127-54.

Wincewicz D, Juchniewicz A, Waszkiewicz N, Braszko JJ. Angiotensin II type 1 receptor blockade by telmisartan prevents stress-induced impairment of memory via HPA axis deactivation and up-regulation of brain-derived neurotrophic factor gene expression. Pharmacol Biochem Behav. 2016 Sep; 148: $108-18$.

Wojtowicz AK, Szychowski KA, Kajta M. PPAR- $\gamma$ agonist GW1929 but not antagonist GW9662 reduces TBBPA-induced neurotoxicity in primary neocortical cells. Neurotox Res. 2014 Apr;25(3):311-22.

Yano M, Matsumura T, Senokuchi T, Ishii N, Murata $\mathrm{Y}$, Taketa $\mathrm{K}$, et al. Statins activate peroxisome proliferator-activated receptor gamma through extracellular signal-regulated kinase $1 / 2$ and p38 mitogen-activated protein kinase-dependent cyclooxygenase- 2 expression in macrophages. Circ Res. 2007 May; 100(10): 1442-51.

Zhao X, Ou Z, Grotta JC, Waxham N, Aronowski J. Peroxisome-proliferator-activated receptor-gamma (PPARgamma) activation protects neurons from NMDA excitotoxicity. Brain Res. 2006 Feb;1073-1074:460-9.

Zou R, He Y, Li YQ, Han M, Ma ZF, Liu XC, et al. Telmisartan protects $5 / 6 \mathrm{Nx}$ rats against renal injury by enhancing nNOS-derived NO generation via regulation of PPAR $y$ signaling. Am J Transl Res. 2014 Oct;6(5):517-27. 